\title{
Cryo-FIB Lamella Milling: A Comprehensive Technique to Prepare Samples of Both Plunge- and High-pressure Frozen-hydrated Specimens for in situ Studies.
}

Miroslava Schaffer ${ }^{1}$, Stefan Pfeffer ${ }^{1}$, Stephan Kleindiek ${ }^{2}$, Julia Mahamid ${ }^{1,3}$, Michael Heymann ${ }^{4}$, Andrew J. Smith ${ }^{2}$, Tim Laugks ${ }^{1}$, Benjamin D. Engel ${ }^{1}$, Sahradha Albert ${ }^{1}$, Wolfgang Baumeister ${ }^{1}$, Juergen M. Plitzko ${ }^{1}$

1. Max Planck Institute of Biochemistry, Dep. of Molecular Structural Biology, Martinsried, Germany

2. Kleindiek Nanotechnik GmbH, Markwiesenstraße 55, Reutlingen, Germany

3. Structural and Computational Biology Unit, European Molecular Biology Laboratory, Heidelberg

4. Max Planck Institute of Biochemistry, Dep. of Cellular and Molecular Biophysics, Martinsried, Germany

In recent years, in situ cryo-electron tomography (cryo-ET) of macromolecules inside cells at subnanometre resolution has become possible through ground breaking developments in sample preparation. Using a cryo-focused ion beam (cryo-FIB) microscope, vitrified specimens are locally thinned to electron transparency, offering cross-sectional views of the sample without introducing preparation artefacts. Such cryo-FIB lamellas can be reproducibly prepared with suitable quality for Volta phase plate contrast-enhanced imaging, enabling in situ studies of membrane-bound macromolecules [1-4].

However, the established cryo-FIB method is only suitable for specimens that can be vitrified in toto by plunge-freezing and are sufficiently small to allow complete removal of material on both sides of the area of interest by ion milling [5]. An interesting challenge is the extension of cryo-FIB sample preparation to high-pressure-frozen (HPF) bulk samples, which would enable studies of tissue or any large macroscopic specimen that can be fully vitrified. Several preparation schemes from materials science, including lamella lift-out with a micro-manipulator needle and in-place bulk-sample H-bar milling, seem promising for adaptation to cryo-preparation of biological samples. However, successful application for cryo-ET at molecular resolution has so far not been shown, possibly due to the stringent sample quality requirements and geometric constraints of the technique.

In this work, we describe a novel cryo-FIB lift-out sample preparation scheme as an integral part of a complete cryo-ET workflow. Utilizing a cryo-adapted micromanipulator 'gripping' tool, which avoids issues of localized material deposition, we selectively extracted fluorescently-labelled volumes of interest from large HPF bulk samples and transferred them onto a customized TEM half-grid for final thinning (Fig. 1). The sample size, thickness and overall quality of the final TEM samples were comparable to the standard lamella-milling approach, enabling high-resolution in situ cryo-ET studies on HPF biological specimens for the first time.

\section{References:}

[1] M Schaffer et al, J Struct Biol. 197 (2017), p. 73.

[2] J Mahamid et al, Science 351 (2016), p. 969.

[3] S Albert et al, PNAS 114 (2017), p. 13726.

[4] YS Bykov et al, eLife 6 (2017), e32493.

[5] M Schaffer et al, Bio Protocol 5 (2015), e1575. 

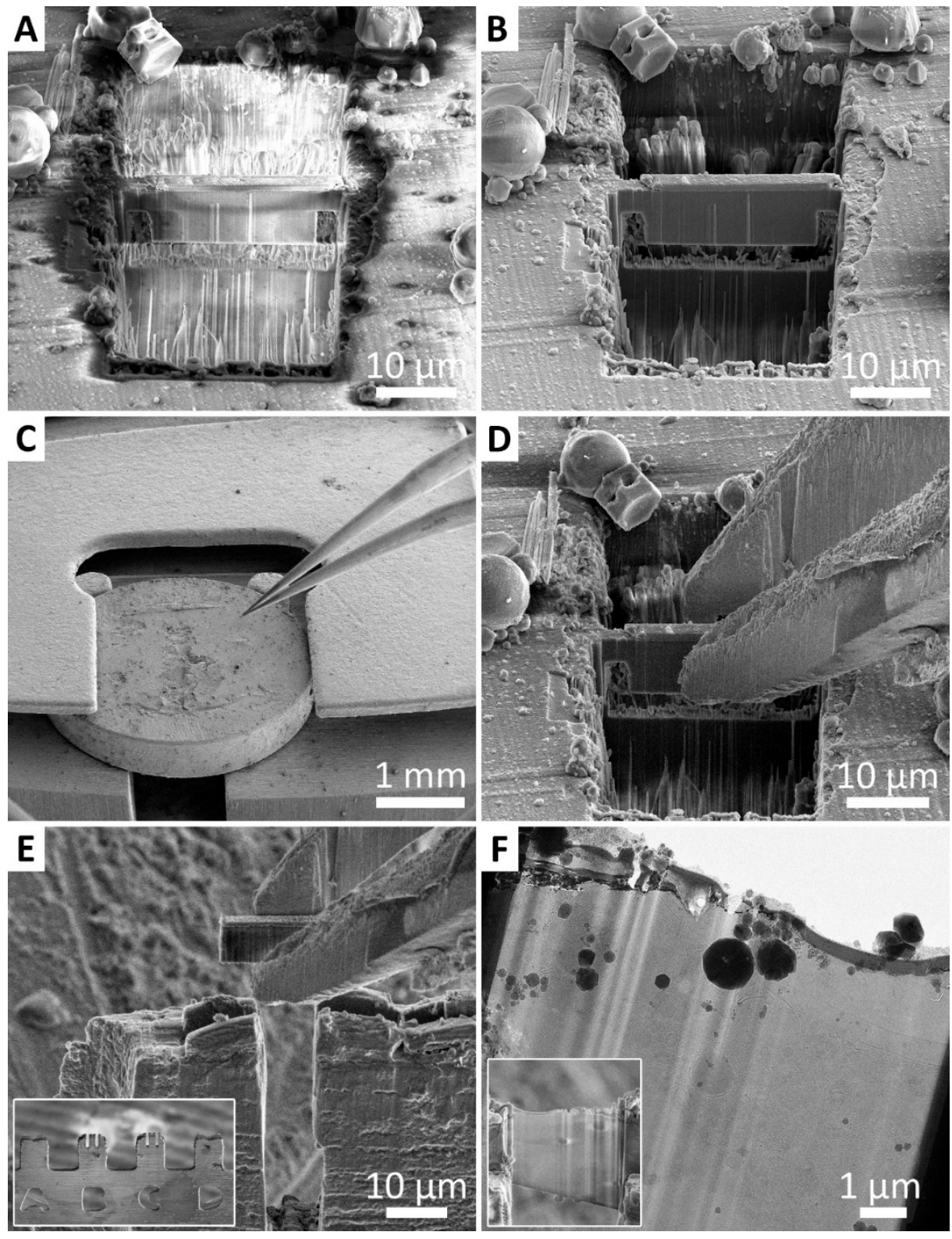

Fig. 1: Cryo-FIB sample preparation utilizing a Kleindiek cryo-gripper micromanipulator. A volume of interest is prepared in an HPF bulk sample by milling two trenches (A) and subsequently under-cutting the volume using an angle such that only two connecting bridges remain (B). The cryo-gripper micromanipulator is inserted (C) and, using a tweezer-like motion, grabs the volume of interest (D), which is then cut loose by the ion-beam milling. The volume is transferred into custom-prepared slits on a TEM half-grid (E) before final thinning to electron transparency. The TEM overview image of the final lamella (F) demonstrates the overall quality of the sample from which cryo-ET data was subsequently acquired and analysed. 